\title{
Nordiques
}

33 | 2017

La transition des villes nordiques : quelles innovations territoriales en périphérie?

\section{Quand Skarphéðinn parle : l'ironie existentielle dans la littérature médiévale islandaise}

\section{Ármann Jakobsson}

Traducteur : Valentin Dezalle

\section{(2) OpenEdition}

\section{Édition électronique}

URL : https://journals.openedition.org/nordiques/3328

DOI : $10.4000 /$ nordiques.3328

ISSN : 2777-8479

Éditeur :

Association Norden, Bibliothèque de Caen la mer

\section{Édition imprimée}

Date de publication : 1 mai 2017

Pagination : 115-127

ISBN : 978-2-9544654-9-4

ISSN : $1761-7677$

\section{Référence électronique}

Ármann Jakobsson, «Quand Skarphéðinn parle : l'ironie existentielle dans la littérature médiévale islandaise », Nordiques [En ligne], 33 | 2017, mis en ligne le 17 janvier 2022, consulté le 02 février 2022. URL : http://journals.openedition.org/nordiques/3328; DOI : https://doi.org/10.4000/nordiques.3328 


\section{Quand Skarphéðinn parle : l'ironie existentielle dans la littérature médiévale islandaise ${ }^{1}$}

Ármann Jakobsson*

\section{RÉSUMÉ}

Une conscience de l'importance de lironie est largement identifiable dans les textes islandais médiévaux, mais n'a fait l'objet que d'un faible nombre de recherches. Cette étude propose une analyse du discours du personnage de Skarphédinn Njálsson et se focalise plus particulièrement sur ses paroles ironiques en avançant la thèse que l'ironie discursive du protagoniste reflète une ironie existentielle qui profile la description du personnage, mais aussi le message de la saga.

\section{ABSTRACT}

Conscious thinking about irony and its importance can be discerned in medieval Icelandic literature and yet this has not been much discussed. In this study, the dialogue of the character Skarphédinn Njallsson is analysed and it is proposed that the irony discernible in his discourse reflects existential irony, a feature significant for the creation of Skarphédinn's character and also for the wider meaning of the saga.

* Ármann Jakobsson est professeur de littérature islandaise médiévale à l'université d'Islande depuis 2011. Il a publié ses recherches en anglais, allemand, danois et suédois.

1 Cet article est initialement paru en islandais dans le no 15.1 (2015) de la revue Ritið publiée aux Presses universitaires d'Islande. Il s'agit ici d'une version écourtée et remaniée par l'auteur et le traducteur. C'est à Régis Boyer qu'on doit l'intégralité des traductions des passages de la Saga de Njáll cités en français, mais à l'auteur de l'article que revient la responsabilité de certains remaniements (N.d.T.). 


\section{QU'EST-CE QUE L'IRONIE ?}

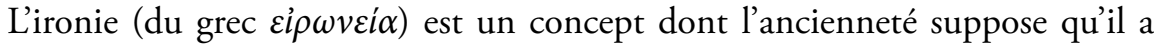
été utilisé de différentes façons et le plus souvent sans définition précise durant une longue période. Il est fondamental que les chercheurs en sciences humaines gardent à l'esprit qu'on faisait généralement un usage limité des normes langagières au cours des siècles passés. De ce fait, il a souvent pu exister une différence de nuance dans la façon d'utiliser des concepts comme celui d'ironie, bien que l'intuition perçue du sens du mot ait été fréquemment fort analogue. Au cours de ces derniers siècles, un assez large consensus a toutefois prévalu en faveur de l'emploi généralisé et de la prédominance de ce concept dans la langue islandaise ${ }^{2}$. L'ironie a été définie comme une figure de style, du discours, comme une forme narrative ou même comme un évènement caractérisé par le décalage entre des attentes et la réalité. La tension entre le sens littéral et le sens profond de l'énoncé a été désignée comme sa caractéristique principale. En outre, on a fait observer que l'ironie présuppose en règle générale un double groupe d'auditeurs qui comprennent ou non le double sens. L'innocence puérile et l'incompréhension de celui qui ne saisit pas le sens constituent les conditions d'une ironie réussie ${ }^{3}$.

Avant que le mouvement romantique ne voie le jour, la version la plus répandue de l'ironie était probablement similaire à celle du prologue de la Saga des rois de Norvège : "Les scaldes ont certes accoutumé de couvrir des plus grandes louanges le personnage devant lequel ils se trouvent, mais aucun d'entre eux n'oserait lui attribuer, en sa présence, des faits dans lesquels toute l'assemblée verrait mensonge et affabulation ; car cela reviendrait à railler et non à glorifier. ${ }^{4}$ En bref : l'hommage est dépourvu de sens ; quelqu'un est couvert de louanges afin d'être tourné en ridicule. On peut lire dans la même veine le célèbre discours de Marc Antoine dans Jules César (1599), où le général ressasse que Brutus et les autres agresseurs de l'empereur sont des hommes d'honneur'. L'ironie prend ensuite une nouvelle

2 Je laisse ici de côté le concept de cynisme et adhère au point de vue de John Haiman (Talk Is Cheap. Sarcasm, Alienation, and the Evolution of Language, Oxford, Oxford UP, 1998, p. 20) qui considère que l'ironie est généralement employée dans le cynisme, mais il s'agit là d'un concept bien plus étendu. Si des circonstances peuvent être ironiques, le cynisme implique toujours, de fait, une personne maniant agressivement l'ironie. Aussi, le cynisme prend chaque fois quelqu'un pour objet, ce qui n'est absolument pas le cas de l'ironie. Suivant cette compréhension des termes, différents exemples d'ironie qui viendront à être mentionnés seront parfois qualifiés de cynisme.

3 Voir notamment Douglas Colin Muecke, The Compass of Irony, Londres, Methuen, 1969, p. 14-39.

4 Snorri Sturluson, Heimskringla. Histoire des rois de Norvège, première partie, trad. de F.X. Dillmann, Paris, Gallimard (L'aube des peuples), 2000, p. 52.

5 William Shakespeare, Jules César, trad. de M. Vinaver [postf. de Richard Marienstras], $2^{e}$ éd., Arles, Actes Sud (Papiers), 1997. Il est ici renvoyé à la scène 2 de l'acte III. Comme souvent, il n'est pas forcément facile d'expliquer brièvement en quoi l'ironie consiste. Antonius avance en tout cas que l'assassinat de César n'a rien d'honorable. Toutefois, on peut être partagé quant à la conclusion à en tirer. Soit Brutus ne peut pas être un homme d'honneur, soit il l'est en général. Dans la dernière alternative, l'assassinat de Jules César devient bien plus ignominieux : une atrocité commise par un homme d'honneur. 
dimension lorsqu'elle est qualifiée d'ironie romantique, bien que le poète romantique et philosophe Friedrich Schlegel (1772-1829) qui est à l'origine de ce concept ne le définît pourtant jamais précisément. Par contre, il est clair que lui et ses épigones considéraient l'ironie comme une gangrène entamant certains textes, voire comme un trait de caractère humain, pas exclusivement comme une figure de style. L'ironie romantique ne vise toutefois pas à atténuer le sens littéral, mais, plutôt, à l'amplifier. Dans l'ironie, comme Schlegel l'explique lui-même dans les Fragments critiques de la revue Lyceum (1797), tout doit être plaisanterie et sérieux, tout doit être présenté à cœur ouvert tout en étant profondément caché6 .

Søren Kierkegaard (1813-1855) alla encore plus loin dans sa thèse de doctorat consacrée à l'ironie intitulée Sur le concept d'ironie constamment rapporté à Socrate (1841) : selon lui, l'ironie n'est pas une simple figure de style, ni même une caractéristique ordinaire d'un texte spécifique, mais la seule attitude capable de permettre de mener cette existence digne que nous qualifions d'humaine. Bien qu'il adhère au point de vue de Georg Wilhelm Friedrich Hegel, qu'il critique l'ironie dans les dialogues socratiques de Platon et qu'il justifie qu'elle soit foncièrement négative, on a signalé que Kierkegaard a néanmoins foi en la force libératrice que l'ironie constitue en pratique, particulièrement si elle est avant tout une attitude à l'égard de la vie et de la réalitét.

On parle généralement de l'ironie existentielle en rapport avec la modernité, comme lors d'un récent débat au cours duquel on se demandait si le débordement excessif d'ironie chez les dénommés "hipsters" ne constituait pas une menace pour la démocratie et la société ${ }^{8}$ ? L'ironie fut pareillement citée en exemple en tant que marqueur de la modernité et comme réponse à la crise axiologique et aux changements sociaux radicaux ${ }^{9}$. Enfin, l'ironie a également joué un rôle dans

6 Voir notamment Douglas Colin Muecke, op. cit., p. 182-215 ; Lars Elleström, Divine Madness. On Interpreting Literature, Music, and the Visual Arts Ironically, Londres - Missisauga - Cranbury N.J., Associated University Presses, 2002, p. 17-20.

7 Voir notamment Andrew Cross, "Neither Either Nor Or : The Peril of Reflexive Irony ", in The Cambridge Companion to Kierkegaard, A. Hannay et G. D. Marino (dir.), Cambridge, Cambridge UP, 1998, p. 125-151. Comme Cross l'explique, cela apparaît encore plus clairement dans la postface de Kierkegaard à son ouvrage précédent intitulé Post-scriptum aux miettes philosophiques (1846).

8 Christy Wampole, "How to Live Without Irony ", New York Time, 17 novembre 2012 ; Jonathan D. Fitzgerald, «Sincerity, Not Irony, is Our Age's Ethos », The Atlantic, 20 novembre 2012. Cette discussion est en fait la réanimation d'une critique influente de l'écrivain David Foster Wallace ( E Unibus Pluram : Television and U.S. Fiction ", Review of Contemporary Fiction, 13, 2, 1993, p. 151-194) dans laquelle certains lecteurs ont clairement identifié le legs kierkegaardien. Aucun des deux auteurs n'est pourtant totalement opposé à l'ironie à laquelle il a été fait référence (voir notamment Allard Den Dulk, « Beyond Endless "Aesthetic" Irony : A Comparison of the Irony Critique of Søren Kierkegaard and David Foster Wallace's Infinite Jest ", Studies in the Novel, 44, 3, 2012, p. 325-345).

9 Ce point a fait l'objet d'une discussion en lien avec la culture islandaise moderne, voir entre autres, Bergljót S. Kristjánsdóttir, "Ég get ekkert sagt : Skáldskapur og hrun », Ritið, 11, 2, 2011, p. 53-66. Bergljót parle notamment d'une confrontation de cadres de pensée, d'un modèle quotidien qui se heurte à un autre cadre qui va démolir le modèle traditionnel. 
les discussions portant sur la littérature islandaise des siècles passés, notamment lorsqu'on se demandait s'il ne serait pas juste de comprendre celle-ci comme une critique implicite de ce qu'elle loue ostensiblement ${ }^{10}$. Pourtant, on compte assez peu de recherches approfondies sur l'ironie dans la littérature médiévale islandaise, hormis l'intéressante analyse de Robert Cook sur le rôle du public dans la Saga de Grettir, dans laquelle il discute à la fois comment l'image du héros est d'abord longtemps plurivoque dans la saga et comment il plane une certaine incertitude quant au caractère positif du personnage ${ }^{11}$. On peut également citer l'interprétation approfondie de la Saga d'Egill proposée par Torfi Tulinius dans laquelle il traite en partie de la plurivocité et des jeux de mots ${ }^{12}$. L'auteur y attire l'attention sur différents genres de plurivocité dans les Sagas islandaises qui suggèrent que leurs auteurs ont été curieux de laisser le public participer à la création du sens du texte ${ }^{13}$. L'ironie intervient évidemment dans de nombreuses interprétations de sagas tant elle est par nature un problème majeur : l'ironie est partout.

On peut ainsi dire que l'ironie présente dans les Sagas islandaises est le plus souvent produite par le discours et par les circonstances. Il est même probable qu'elle soit existentielle, comme nous l'exposerons dans la présente étude. Malgré la domination d'un vif intérêt pour les personnages des Sagas islandaises et leurs discours, on compte toutefois peu d'écrits scientifiques portant sur ce sujet hormis cette tentative que nous faisons ici. Nous allons effectivement nous concentrer sur le discours direct d'un seul protagoniste en nous focalisant particulièrement sur l'ironie dans ses paroles, après quoi nous nous demanderons si l'ironie linguistique ne pourrait pas refléter une ironie existentielle qui profilerait la description du personnage, mais aussi le message de la saga.

\section{LE DISCOURS DE SKaRPHÉĐINN}

Skarphéðinn Njálsson est un des personnages les plus connus de la tradition des Sagas islandaises. Pourtant, il est exclusivement mentionné dans la Saga de Njáll le brûlé, à l'exception peut-être du Livre de la colonisation ${ }^{14}$. Cela ne signifie pas qu'aucun homme de ce nom n'a existé du temps des sagas des Islandais,

10 Voir notamment Njörður Njarðvík, "Laxdæla saga - en tidskritik », Arkiv för nordisk filologi, 86, 1971, p. 72-81 ; Robert G. Cook, "Women and Men in Laxdæla Saga », Skáldskaparmál, 2, 1992, p. 34-59. Ce sont des analyses assez différentes, mais qui font toutefois chacune entrer en ligne de compte l'attitude critique des historiens vis-à-vis des personnages et du contenu des sagas qu'il ne faut pas nécessairement prendre au pied de la lettre.

11 Robert G. Cook, «The Reader in Grettis Saga », Saga-Book, 21, 1984-1985, p. 133-154.

12 Torfi Tulinius, Skáldið i skriftinni. Snorri Sturluson og Egils saga, Reykjavík, Hið íslenska bókmenntafélag og Reykjavíkur Akademían, 2004, en particulier p. 117-125.

13 Ármann Jakobsson, "Some Types of Ambiguities in the Sagas of the Icelanders ", Arkiv for nordisk filologi, 119, 2004, p. 37-53.

14 Brennu-Njáls saga, in Íslenzk fornrit, XII, E. Ó. Sveinsson (éd.), Reykjavík, Hið íslenzka fornritafélag, 1954, p. 71, notes 1 et 3 . 
c'est-à-dire vers 930-1030 de notre ère en Islande, et encore moins une tradition narrative concernant Skarphéðinn au Moyen Âge qui ait été connue et employée par l'auteur de la Saga de Njáll ${ }^{15}$. Toutefois, l'existence de Skarphéðinn au siècle des sagas ou bien une ancienne tradition narrative le concernant ne nous interdit pas d'utiliser la notion de création de personnage à propos de son rôle dans la Saga de Njáll, quoiqu'on pourrait d'ailleurs tout aussi bien avoir recours à celle d'interprétation de personnage lorsque des auteurs composent un personnage de fiction en s'inspirant de quelqu'un de réel, qu'ils soient historiens ou poètes. Il existe évidemment une différence entre l'interprétation ou la création d'un personnage par le poète et par l'historien - par exemple une différence en termes de liberté que le poète et l'historien s'imaginent avoir d'interpréter le personnage -, mais la création d'un personnage dans les écrits historiques suit exactement le même procédé que dans les écrits romanesques ${ }^{16}$.

La caractérisation d'un personnage est une notion complexe. Nous savons tous que la caractérisation directe n'en constitue qu'une partie, quoiqu'elle ait tendance à être légèrement écrasante dans les écrits scientifiques portant sur les caractérisations de personnages dans les sagas. Skarphéðinn est véritablement décrit de façon directe lorsqu'il est introduit au chapitre 5 de la Saga de Njáll dans lequel il est à la fois question de son apparence, de ses dispositions et de ses caractéristiques. On nous dit notamment : " Qu'il parle à propos et rapidement, quoiqu'il soit modéré la plupart du temps ${ }^{17}$. Comme souvent dans les textes littéraires complexes, il est avant tout caractérisé indirectement par ses paroles et par ses actes. Il a depuis longtemps été communément admis par les chercheurs étudiant les Sagas islandaises que de telles caractérisations de personnages n'ont pas moins de valeur que les caractérisations plus formelles suivant l'introduction d'un nouveau protagoniste dans une saga ${ }^{18}$. Au demeurant, on a rarement cherché à se

15 La notion d'auteur de la Saga de Njáll ne fait ici référence qu’à l'auteur dans l'œuvre ou aux significations premières du texte. Il n'est en rien question d'un Islandais ou d'Islandais qui vivaient spécifiquement au XIII siècle.

16 Bien que les Sagas islandaises ne soient pas des romans, elles ont une forme commune avec les romans historiques, telle qu'on l'a bien souvent indiqué, voir notamment Joseph Harris, « Saga as Historical Novel ", in Structure and Meaning in Old Norse Literature. New Approaches to Textual Analysis and Literary Criticism, J. Lindow, L. Lönnroth et G. W. Weber (dir.), Odense, Odense UP, 1986, p. 187-219.

17 Saga de Njáll le Brûlé, trad. Régis Boyer, Paris, Gallimard, 1987, p. 1242. Toutes les traductions de fragments de textes des Sagas islandaises apparaissant dans cet article sont de Régis Boyer. Toutefois, certaines d'entre elles sont parfois légèrement modifiées à la demande de l'auteur (N.d.T.).

18 Comme l'énonce Vésteinn Ólason : «Les personnages importants sont souvent introduits dans une saga par de courtes ou même longues références portant sur leur apparence, leurs dispositions et leurs vertus. [...] Les hommes s'illustrent toutefois avant tout eux-mêmes par leurs paroles et leurs faits, ainsi que par la conséquence de joindre le geste à la parole " (Vésteinn Ólason, Samraður við söguöld. Frásagnarlist Íslendingasagna og fortíðarmynd, Reykjavík, Heimskringla, háskólaforlag Máls og menningar, 1998, p. 109). 
pencher de plus près sur le discours individuel d'un personnage des Sagas islandaises tel que nous tentons de le faire $\mathrm{ici}^{19}$.

Skarphéðinn prend pour la première fois la parole au milieu de l'épisode des "meurtres des domestiques ", après que Bergpóra a embauché le mystérieux laboureur Atli à Bergpórshvoll et lui a notamment assigné la tâche d'entretenir le conflit avec Hliðarendi ${ }^{20}$. Njáll répond à cela en disant qu'Atli sera très productif, mais qu'il ignore combien il sera bienfaisant ${ }^{21}$. Une référence à l'amitié de Skarphéðinn pour $\mathrm{Atli}^{22}$ est aussi glissée. Skarphéðinn demande ensuite à Njáll avec quel argent ce dernier se rend au Parlement, à quoi son père répond qu'il s'agit de la compensation versée pour la mort de Svartur. Skarphéðinn prend alors pour la première fois la parole et dit : "Cela servira bien à quelque chose ", qui est suivi de « et ricana $»^{23}$. On a bien des fois montré que le ricanement de Skarphéðinn est chargé de sens et parfois même associé à de l'indépendance obstinée ${ }^{24}$. Plus récemment, certains ont aussi trouvé assez tentante l'idée de rapprocher le ricanement de désordres mentaux de différents types. Rien d'étonnant à cela quand

19 Compte tenu de l'universalité de l'ironie dans la langue (et dans le monde), nous ne parlerons pas du contexte particulier de ce texte du XIII e siècle ou de son sens pour le public de cette époque. Différents ouvrages en traitent, notamment Lars Lönnroth, Njáls Saga. A Critical Introduction, Berkeley, University of California Press, 1976 ; Hermann Pálsson, Uppruni Njálu og hugmyndir, Reykjavík, Menningarsjóður, 1984.

20 Rien n'est dit à propos d'Atli et de sa vie avant et après qu'il s'est présenté lui-même à son arrivée à cheval à la ferme de Bergpórshvoll (Saga de Njáll le Brûlé, op. cit., p. 1258-1259). Il dit être : 1) sans toit, 2) laboureur, 3) de mauvais caractère, 4) la cause de nombreuses blessures à panser, 5) désireux d'être élevé au rang d'homme libre. En outre, il dit être comme Kolur, l'intendant de Hallgerður, une personne malintentionnée (Saga de Njáll le Brûlé, op. cit., p. 1259). Le public doit toutefois prendre avec réserve la description qu'un homme fait ainsi de lui-même, aussi insolite que cela puisse paraitre. Gunnar de Hlíðarendi assure qu'il n'est pas d'hommes plus viril qu'Atli. Ses actes nous laissent deviner qu'il est vaillant, qu'il ne craint pas la mort et qu'il n'est pas dépourvu de profondeur d'esprit, comme en témoignent aussi ses célèbres paroles prononcées au moment où il tue Kolur : «Il ne te reste plus que le plus difficile " (à quoi est ajouté dans le Reykjabók, " et c'est à mourir » [ibid.]).

21 Njáll recourt ici à différentes figures de style avec beaucoup d'adresse, notamment l'ironie. On peut également remarquer une antithèse, une épiphore, un isocolon et des litotes.

22 Tel que Richard F. Allen l'a justement indiqué, cela consiste en une description particulière d'Atli (Fire and Iron. Critical Approaches to Njáls Saga, Pittsburgh, University of Pittsburgh Press, 1971, p. 107). Donné que Skarphéðinn n'est pas apparu un grand nombre fois dans la saga sur ces entrefaites, il faut garder à l'esprit que cette description constitue également un message important à propos de Skarphéðinn lui-même, tout comme l'amitié de Práinn Sigfússon et de Víga-Hrappur qui nous donne plus tard des renseignements significatifs sur leurs personnalités (pour plus de détails, voir : Ármann Jakobsson, "The Impetuousness of Práinn Sigfússon : Leadership, Virtue and Villainy in Njáls Saga ", Arkiv för nordisk filologi, 124, 2009, p. 53-67).

23 Saga de Njáll le Brûlé, op. cit., p. 1259.

24 Voir notamment Ai Low Soon, «The Mirthless Content of Skarphéðinn's Grin », Medium Aevum, 65, 1997, p. 101-108 ; Kirsten Wolf, "Laughter in Old Norse-Icelandic Literature ", Scripta Islandica, 51, 2000, p. 93-117. Skarphéðinn ricane en tout neuf à dix fois dans la saga. 
on sait comment la médecine forme la compréhension du monde de l'homme moderne $\mathrm{e}^{25}$.

Mais si nous nous concentrons d'abord sur le sens évident du ricanement, il est plus commode de conclure provisoirement que Skarphéðinn s'amuse. Il est naturel de se demander dans la foulée si sa réplique est ironique et comment il va nous falloir la déchiffrer. Ce qui caractérise principalement l'ironie est la facilité de son identification. Expliquer définitivement ce en quoi elle consiste est en revanche une tâche beaucoup plus ardue. Dans le cas présent, elle porte sur la connaissance commune de la suite du conflit dont Skarphéðinn s'abstient de parler ; il confirme au contraire la conspiration langagière et la solidarité sociétale entre père et fils par le non-dit ${ }^{26}$. La phrase signifie littéralement qu'un bénéfice peut être tiré de l'argent, alors que le ricanement suggère qu'il devine déjà ce que cette somme vaudra au bout du compte.

Lorsqu'on passe en revue les prises de parole de Skarphéðinn dans l'ensemble de la Saga de Njáll le Brûlé, on peut trouver quelques exemples d'ironie que j'exploite ici brièvement non seulement pour argumenter la thèse selon laquelle le discours de Skarphéðinn est imprégné d'ironie, mais aussi pour montrer par la même occasion combien d'aspects différents elle revêt et combien elle ne se laisse pas expliquer au moyen de formules toutes faites qui prouveraient que quelque chose nous a échappé à un moment donné.

Chapitre 7 : Après le meurtre de Kolur par Alti, Skarphéðinn rapporte que " les esclaves sont beaucoup plus entreprenants qu'auparavant ; jusqu'ici, ils se bagarraient entre eux, et il n'y a rien à redire à cela ; mais maintenant, voilà qu'ils se mettent à s'entretuer ». Puis il ricane. En parlant de la sorte, Skarphéðinn se distancie du cours des évènements en faisant comme si les meurtres des

25 L'idée selon laquelle Skarphéðinn est un genre de malade mental a été populaire aux XXe et $\mathrm{XXI}^{\mathrm{e}}$ siècles et a notamment été avancée par le poète Hans E. Kinck, Sagaenes ånd og skikkelser, Oslo, Aschehoug, 1951, p. 28 (paru à l'origine dans Mange slags kunst [1921] qu'on peut dater de 1916). Kinck considère le ricanement de Skarphéðinn comme un très sérieux indicateur et Ai Low Soon est du même avis (voir note 31). William I. Miller n'exagère pas autant, mais tient toutefois le ricanement pour un genre de manque de contrôle de soi : "Skarphédinn tries to control his feelings with a bantering tone and with a grin. Skarphéðinn's grins are always a bit unnerving. His grin suggest sadistic urgings and hostile sensation at the same time that it suggests desperate efforts at self-control. So the grin is multiply determined. At some level it means to cover up and control, at another it means to reveal, and at yet another it can't be meant at all because it appears involuntarily" ("Emotions and the Sagas ", in From Sagas to Society. Comparative Approaches to Early Iceland, G. Pálsson (dir.), Enfield Lock, Hisarlik Press, 1992, p. 89-109, ici p. 101.) Cela est assez juste, mais il ne me semble pourtant pas exclu que le ricanement soit plus réfléchi qu'ils ne considèrent tous les deux, c'est-à-dire que Skarphéðinn ricane sciemment et que le sadisme ne s'y trouve pas mêlé (sauf s'il a peut-être sa place dans l'ironie en général, ce que nous discuterons plus en détail en fin d'article).

26 William I. Miller dit : "Njal and Skarphedin are both here making an in-the-know joke, registering their knowledge of the paradox of buying peace " "Why is Your Axe Bloody?". A Reading of Njáls Saga, Oxford, Oxford UP, 2014, p. 81). 
domestiques étaient semblables à n'importe quelle autre plaisanterie - une échauffourée qui a un faux air d'homicide. Il s'agit là d'un bon exemple d'ironie produisant le même effet que celle que Kierkegaard décrit chez Socrate. Skarphéðinn prend de la hauteur et se soustrait du même coup au conflit de la saga, ce qui est bien sûr fondamentalement négatif du point de vue moral - Skarphéðinn se permet ainsi de se libérer de toute obligation vis-à-vis de son entourage ${ }^{27}$. Une grande partie des différents auditeurs historiques a dû pourtant rire avec Skarphéðinn et trouver ce dernier bien plus sympathique du fait de son humour et peut-être même le percevoir comme un spectateur qui prend place, en quelque sorte, en dehors de l'œuvre et donc au même endroit qu'eux. Il est aussi possible que ce commentaire de Skarphéðinn, tout comme de nombreuses autres remarques du personnage, serve aux mêmes fins que celles du "comic relief» dans les tragédies de Shakespeare : soulager le lecteur des sentiments intenses qu'un homicide a suscités par ailleurs ${ }^{28}$. Au chapitre 38 , il réitère le même type d'observation : "Hallgerður ne laisse pas nos domestiques mourir de vieillesse ", ce qui est un bon exemple de litote à des fins humoristiques et montre comment Skarphéðinn est non seulement drôle, mais également un dur-à-cuir, un homme froid restant de marbre face à un différend insignifiant comme le meurtre de domestiques ${ }^{29}$.

Chapitre 44 : Le passage où Bergpóra provoque ses fils en leur racontant la boutade scatologique d'Hallgerður constitue une des pépites ironiques de la saga. Skarphéðinn réplique en ces termes : "La vieille, notre mère, s'amuse " ("à nous irriter » est ajouté dans un manuscrit). Il ricane de nouveau, mais transpire et se couvre aussi de taches rouges aux joues. C'est ainsi que l'ironie dramatique est produite : l'insouciance faisant jour dans son discours ne témoigne pas de son véritable état d'âme. Skarphéðinn mentionne également ne pas avoir un « caractère

27 Les lecteurs attentifs verront peut-être tout de suite une certaine ressemblance entre son attitude et celle de M. Bennett dans Orgueil et Préjugés (1813) de Jane Austen. M. Bennett représente exactement comment une attitude ironique peut rendre des personnages littéraires plus sympathiques $\left(\mathrm{M}^{\mathrm{me}}\right.$ Bennett est en revanche dépourvue d'ironie et n'égale en rien sa sympathie). Toutefois, M. Bennet pourrait également être accusé de fuir, de ne pas observer les usages et d'être ainsi peu utile à l'action essentielle de l'œuvre.

28 Voir notamment Frederic B. Tromly, « Macbeth and His Porter ", Shakespeare Quaterly, 26, 1975, p. 151-156. Il ne s'agit toutefois pas de la seule méthode employée dans le texte à cet effet ; on peut également signaler que la plupart des valets de ferme sont introduits dans la saga à seule fin d'être tués et qu'ils constituent avant tout plus ou moins des stéréotypes, en particulier les deux premiers, Kolur et Svartur. On peut ainsi présupposer une certaine distance ironique du public par rapport à ces meurtres inauguraux qui ont pour effet de rapprocher la position de l'audience de celle de Skarphéðinn dans le récit ; exactement comme s'il les y avait mêlés petit à petit.

29 Il faut en effet souligner la grande modernité de l'utilisation du terme « cool » à propos d'un durà-cuir, puisque la fraîcheur n'a commencé à entrer en grâce en tant que caractéristique de la sérénité et du flegme qu'au XX $X^{\mathrm{e}}$ siècle, voir Peter Stearns, American Cool. Constructing a Twentieth-Century Emotional Style, New York, New York UP, 1994. Par contre, le flegme est une vertu qui est célébrée au Moyen Âge. Elle est donc hautement considérée dans la Saga de Njáll le Brûlé ainsi que dans divers autres écrits islandais médiévaux. 
de femme " pour se fâcher de tout, mais au vu de la transpiration et des taches, il se met bien en colère, ce qui renforce l'ironie circonstancielle : celui qui prétend ne pas se mettre en colère se fâche pourtant bien - et s'est donc lui-même considéré être une femme ${ }^{30}$. Bien que les paroles de Skarphéðinn soient essentiellement du même genre que celles de ses précédentes déclarations, il tente encore de se distancier, voire de se tenir en dehors des évènements à la façon d'un juge cynique. Tous les personnages savent que Bergpóra est tout à fait sérieuse et n’a pas ici le mot pour rire ; mais c'est en faisant des simagrées que Skarphéðinn essaie de minimiser la véritable gravité des évènements. Des signes extérieurs montrent toutefois l'échec de la manœuvre et indiquent alors que Skarphéðinn est devenu un acteur même si son discours continue d'être caractérisé par l'ironie. Cela laisse porter à croire que l'ironie constitue pour lui un genre de bouclier ou d'armure, tel que l'a suggéré Einar Ólafur Sveinsson ${ }^{31}$, et il nous revient de ne pas sous-estimer ce rôle fondamental de l'ironie dans un monde aussi cruel que celui des Sagas islandaises. En conséquence, on peut dire qu'il n'est absolument pas certain qu'il soit impartial ou distant dans son for intérieur et cela constitue peut-être ici un bon exemple de cette libération qui consiste en l'attitude ironique : elle simplifie son rôle d'homme équilibré et détendu alors qu'il bouillonne de sentiments ${ }^{32}$. Comme nous le verrons plus tard, on pourrait éventuellement y voir une fuite de la réalité, mais la question est un brin plus complexe que cela.

Chapitre 5: "Voyez-vous l'elfe rouge ? " dit Skarphéðinn aux autres fils de Njáll à la vue de Sigmundur en habits colorés. Einar Ólafur Sveinsson appelle cela "de l'ironisation construite à la façon de svartálfr, elfe noir " ${ }^{33}$, ce qui nous avance assez peu sur le fond de cette histoire. On ignore pour quelle raison álfur aurait été employé comme un terme de dénigrement à cette période, même si ce fut plus tard le $\operatorname{cas}^{34}$. Skarphéðinn pourrait au contraire se référer ici au statut supérieur des elfes qui disposent de plus de pouvoirs que les hommes et leur sont supérieurs. Les habits colorés étaient jugés très élégants, ce qui fait qu’il était possiblement encore plus chic d'être un elfe en habits colorés, mais lorsque Skarphéðinn fait ce type de remarque sur le style vestimentaire de Sigmundur, il se pourrait que ce

30 Il se pourrait que ce soit surtout en raison de ce détail que les interprétateurs ont incliné à comprendre Skarphéðinn comme une personne particulièrement émotive à la suite de l'analyse d'Einar Ólafur Sveinsson (voir note 31).

31 Einar Ólafur Sveinsson, Á Njálsbúð. Bók um mikið listaverk, Reykjavík, Hið íslenzka bókmenntafélag, 1943, p. 116. Il n'emploie pas le concept d'ironie mais « de négligence et de dérision » et considère que la dérision recouvre « les simagrées, l'humour noir, l'amertume, l'acerbité enflammée, la verte semonce ".

32 C'est effectivement un motif narratif de la saga et exactement la même tension sentimentale et flegmatique que l'on peut rencontrer plus tard dans la saga chez le personnage de Flosi de Svínafell qui ne pratique toutefois pas l'ironie afin de se distancier, comme le fait Skarphéðinn.

33 Brennu-Njáls saga, op. cit., p. 115, note 7.

34 Voir Jón Ârnason, İslenzkar pjóðsögur og afintýri, Leipzig, J. C. Hinrichs, 1862, t. I, p. 1 : « Mais l'elfe que nous mentionnons maintenant prête foncièrement à sourire. » 
soit afin de le tourner en dérision tout en lui rendant l'hommage, plus ou moins comme lorsque nos contemporains appellent quelqu'un de lent "une flèche " tout en voulant dire le contraire. L'expression elfe rouge suscite simultanément une association de pensées à propos du décalage entre le bel habit de Sigmundur et les atrocités qui vont bientôt se dérouler, et met alors peut-être également en évidence l'opposition sémantique entre la couleur rouge de l'habit et celle du sang que Skarphéðinn pense verser.

Chapitre 91 : Skarphéðinn et ses frères se rendent à Grjótá afin d'entrer en controverse avec Práinn et ses hommes. Lorsqu'ils arrivent, les gens de la maison réagissent de telle façon qu'aucun d'eux ne les salue et Skarphéðinn de dire : "Bienvenue à nous tous ». Il n'est pas précisé s'il ricane cette fois-ci, mais ses paroles sont indéniablement lourdes de sens. C'est l'un de ses commentaires ironiques les plus achevés et peut-être le plus influent du fait de sa banalité. Ici, il s'agit sensiblement du même genre d'humour que lorsqu'un de nos contemporains dit lui-même "merci " à quelqu'un qui a oublié de remercier et attire ainsi l'attention sur son impolitesse. Par comparaison, les paroles suivantes de Skarphéðinn, adressées à Hallgerður, insinuent qu'elle est ou bien une femme qu'on met au coin ou bien une putain et relèvent bien plus de la franche brusquerie que de l'ironie et sont ainsi beaucoup plus grossières que son humour ordinaire.

Chapitre 119-120 : On ne reviendra pas ici en détail sur les évènements relatés dans ces chapitres, mais Skarphéðinn a une joute verbale avec le plus éminent de tous les chefs d'Islande et remporte la victoire. C'est peut-être ici et pendant le meurtre de Práinn que Skarphéðinn culmine dans son art. On peut voir différents exemples d'ironie dans ces échanges verbaux qui ont la caractéristique secondaire d'être de vertes injures fondées sur des commérages qui devaient être alors extrêmement connus et brocardaient chacun des chefs islandais ${ }^{35}$. Les paroles les plus mémorables sont toutefois celles de Skarphéðinn lorsqu'il finit par demander à Ásgrímur Elliða-Grímsson où il faut qu’il aille. Quand ce dernier répond qu'il doit se rendre dans son propre baraquement, Skarphéðinn réplique en disant : "Alors, c'est que nous en avons assez d'aller quémander dans les baraquements. » Après tous ces échanges tendus, on peut considérer cette phrase comme du " comic relief» et un bon exemple de l'utilisation que Skarphéðinn fait de l'humour afin de prendre de la hauteur par rapport au tourbillon des évènements. Lironie tient ici à ce qu'il ne dit rien d'autre que ce qui lui saute aux yeux. En restant en retrait, il observe de haut le cours des évènements, ainsi que son propre destin, et évite

35 Voir notamment Ármann Jakobsson, «Some Types of Ambiguities in the Sagas of the Icelanders », op. cit., p. 38-41. On y renvoie au paradoxe que les chefs d'Islande de plus haut rang ne reconnaissent pas tout de suite Skarphéðinn à l'Alping et cet article traite aussi de la question de son sens. Mais nous ne discuterons pas ici des paradoxes. 
de s'affliger de ce qui est advenu. Il est parvenu de la sorte à faire simultanément figure de tête d'affiche, de critique dramatique, de personnage et de spectateur.

Chapitre 23 : Skarphéðinn reste longtemps fort taciturne après l'échec de la conciliation à l'Alping. On nous le décrit d'abord debout, silencieux et ricanant, puis se passant la main sur le front en ricanant de nouveau. À la fin, il se tient seul sur le banc du milieu tandis que les autres se trouvent soit à l'est soit à l'ouest de la Lögrétta ${ }^{36}$. De la sorte, il reste encore de nouveau comme en retrait visà-vis du cours des évènements sur lesquels il se focalise ensuite lorsqu'il jette à Flosi des braies bleues de femme et l'appelle la mariée du génie de Svínafell. En l'occurrence, la distance ironique est physique, mais pas discursive. Le ricanement délivre un autre message que ceux qui sont transmis verbalement. Comme il a déjà été indiqué, l'auteur de la Saga de Njáll fait du ricanement de Skarphéðinn le symbole de la distance ironique ${ }^{37}$.

Chapitre 29: Au milieu de l'épisode du supplice de Njáll par le feu, ce dernier est endormi lorsque Skarphéðinn lui fait ses adieux en disant : « Notre père va se coucher de bonne heure, et il fallait s'y attendre : c'est un vieil homme. » Ces mots prennent une tournure ironique du fait du contexte. Un vieillard n'est pas ici en train de se coucher pour s'octroyer un moment de répit, mais se reposer pour l'éternité - le banal et le quotidien sont de nouveau opposés au monstrueux et à l'ultime. Skarphéðinn fait comme s'il s'agissait d'une soirée semblable à une autre et feint de ne pas être affecté par l'énormité des évènements. Il n'apparaît nulle part ailleurs qu'ici aussi limpidement que l'ironie est sa façon de s'éloigner d'une réalité désagréable en plus d'atteindre une sérénité surhumaine et de conserver un calme stoïque face à son destin.

Comme on peut clairement le voir dans cette étude relativement succincte s'appuyant sur quelques exemples, le personnage de saga Skarphéðinn est principalement construit par ses propres paroles et par l'ironie caractérisant son discours. Il parvient ainsi fréquemment à sortir du drame de la saga pour prendre place dans le public où les auditeurs de la saga sont eux-mêmes assis et où il est fort possible qu'ils compatissent avec lui ${ }^{38}$, car il va de soi que les personnages amusants sont naturellement plus sympathiques que ceux qui sont dépourvus

36 Voir notamment à propos de cette scène : Ármann Jakobsson, « Masculinity and Politics in Njáls saga », Viator, 38, 2007, p. 191-215, en particulier p. 196-201.

37 Miller dit joliment : "The grin unnerves everyone, as well it should; it is the embodiment of the ironic principle that threatens purpose with ultimate meaninglessness " (Why is Your Axe Bloody? "..., op. cit., p. 209). C'est néanmoins peut-être un peu exagéré ici. Ne pourrait-on pas interpréter plutôt la signification du ricanement ironique exactement de la manière suivante : le ricaneur ne se soumet pas à la signification que d'autres ont donné à l'évènement et démoli du même coup le sens véritable. Il ne le fait toutefois pas nécessairement afin de le remplacer par un vide sémantique.

38 Et inversement, car avec son ironie continuelle, Skarphéðinn fait aussi montre d'une certaine solidarité avec le public de la saga. 
d'esprit. L'ironie est en même temps sa manière de se distancier des évènements de la saga - et il est donc facile d'interpréter la distance ironique comme un genre d'échappatoire - et d'atteindre la sérénité qui l'aide à faire preuve de courage dans les moments difficiles. De ce fait, le discours ironique de Skarphéðinn lui donne l'impression d'être un dur - l'ironie consiste en cette certaine âpreté de l'homme qui garde ses distances et ne s'abandonne pas à ses sentiments.

\section{SKARPHÉĐINN ET L'EXISTENCE}

La distance ironique de Skarphéðinn est loin d'induire le public à le concevoir comme un homme insensible, les taches rouges ont bien servi l'auteur à faire savoir qu'il est également doué de sentiments, et Skarphéðinn parvient souvent à être aigu et délicat dans son discours ironique, surtout dans ses paroles d'adieu au vieil homme précocement assoupi dans l'incendie.

L'ironie témoigne aussi d'un certain raffinement chez Skarphéðinn. Même si ce dernier est un novice qui n'a connu aucune réussite particulière, qui n'est jamais parti d'Islande où il se tient d'ailleurs constamment dans l'ombre de son père, Skarphéðinn possède un bon nombre des meilleures dispositions du courtisan, parmi lesquelles on compte notamment la gaieté et l'ironie. Bien qu'il soit connu pour des meurtres, il est aussi loin d'être frustre ou sauvage. Aussi fidèle à son père qu'un bon courtisan à son roi, il est par-dessus tout et le plus souvent d'un tempérament flegmatique. Skarphéðinn apparaît comme un homme étonnamment raffiné et mondain, mais d'une très grande intégrité. Il se tient le plus souvent à une même règle et reste égal à lui-même en toutes circonstances et ainsi dépourvu de duplicité malgré son art ironique. C'est peut-être là un point fondamental dans la compréhension de l'ironie : elle n'a pas besoin de signifier mauvaise foi ou fourberie, ni même fuite. L'ironie lui concède évidemment une certaine distance par rapport à ce monde et ce destin abominables qui lui sont créés, mais cette distance lui est nécessaire afin de rester égal à lui-même dans un monde rude - ce qui est peut-être le cas pour l'homme en général. On pourrait également envisager sous cet éclairage l'idée répandue selon laquelle l'ironie est bien plus caractérisée par la cruauté que l'association ordinaire de la violence et du rire. Est-ce que Skarphéðinn est un sadique du fait qu'il ricane et fait preuve d'ironie à l'égard de la violence ? Ou bien ne s'agit-il que d'une manière de faire face à l'âpreté et à la douleur du monde ? On peut se demander par la même occasion si la distance ironique n’implique pas une critique de la société spécifique dans laquelle Skarphéðinn est intégré ou si elle n'est pas plutôt existentielle par principe. Encore, il serait du même coup intéressant de mettre à jour les autres moyens existants dont 
dispose celui qui cherche une issue à ce monde difficile avec lequel il faut que l'homme poursuive sa lutte ${ }^{39}$.

Tel qu'on l'a précédemment mentionné, l'ironie fonde cette double solidarité entre Skarphéðinn et le public de la saga. L'audience est acquise à lui là où il se conduit comme un auditeur. En retour, il fait preuve de respect et de solidarité envers ceux qui écoutent la saga. Le personnage (comme représentant de l'auteur) prend part au complot avec les auditeurs, ce qui a l'effet paradoxal de nous rapprocher et de nous distancier à la fois de l'histoire. En se tenant en dehors d'elle, Skarphéðinn fait appel à notre sympathie et nous entraîne de la même façon dans le récit. De ce fait, nous conservons un double point de vue sur l'histoire, à la fois interne et externe.

L'analyse du discours de Skarphéðinn et de son ironie montre clairement comment ce célèbre personnage des Sagas islandaises - qui est non seulement un héros, mais aussi un "punk " de son temps ayant aussi bien réussi à séduire le public des $\mathrm{XX}^{\mathrm{e}}$ et $\mathrm{XXI}^{\mathrm{e}}$ siècles que celui du Moyen Âge - est en vérité construit par son propre discours ironique ${ }^{40}$. Il est à la fois patent que son ironie n'est pas exclusivement une figure de style ou une forme discursive, mais constitue plutôt une réaction face à un danger de mort, une ironie existentielle qui est à bien des égards exemplaire de cette caractéristique de l'ironie : celle de servir l'individu à être présent à deux endroits à la fois, à l'intérieur et en dehors de sa propre existence. La création et la réception de ce personnage constituent pour l'auteur et le lecteur l'occasion d'exécuter une chorégraphie qui montre bien quel degré de raffinement les Sagas islandaises peuvent atteindre. Le personnage de Skarphéðinn est non seulement lui-même, mais également un reflet du public de la saga qui peut affronter, à travers lui, sa propre destinée et trouver des moyens de lutter contre elle.

\section{Traduit de l'islandais par Valentin Dezalle}

39 Qui sait sinon le poète atomiste Steinn Steinarr si ce dernier n'avait pas distingué l'ironie existentielle du personnage de Skarphéðinn lorsqu'il dit que celui-ci a cherché « une issue dans l'effroi abattu d'un homme mourant » ("Skarphéðinn í brennunni », in Ljóð, Reykjavík, Heimskringla, 1937, p. 10)?

40 Le parallèle avec le punk consiste notamment en l'opposition relativement passive de Skarphéðinn qui se distancie des évènements au lieu de se soulever directement, bien qu'il n'aille pas aussi loin que les punks des années 1970 qui se mettaient en scène comme des victimes. 